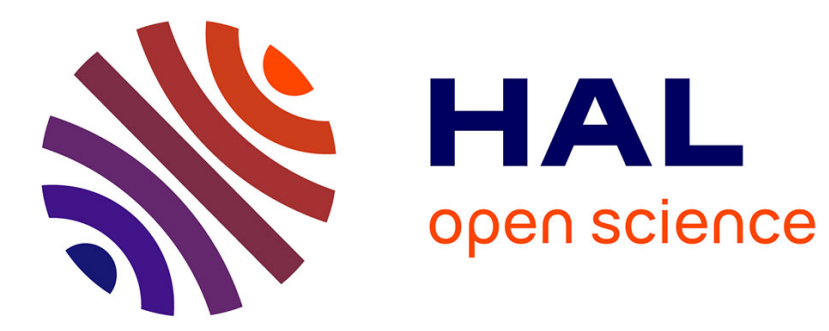

\title{
An a-contrario approach to quasi-periodic noise removal
}

Frédéric Sur

\section{To cite this version:}

Frédéric Sur. An a-contrario approach to quasi-periodic noise removal. ICIP - IEEE International Conference on Image Processing, Sep 2015, Québec City, Canada. 10.1109/ICIP.2015.7351524 hal01211397

\section{HAL Id: hal-01211397 \\ https://inria.hal.science/hal-01211397}

Submitted on 5 Oct 2015

HAL is a multi-disciplinary open access archive for the deposit and dissemination of scientific research documents, whether they are published or not. The documents may come from teaching and research institutions in France or abroad, or from public or private research centers.
L'archive ouverte pluridisciplinaire HAL, est destinée au dépôt et à la diffusion de documents scientifiques de niveau recherche, publiés ou non, émanant des établissements d'enseignement et de recherche français ou étrangers, des laboratoires publics ou privés. 


\title{
AN A-CONTRARIO APPROACH TO QUASI-PERIODIC NOISE REMOVAL
}

\author{
Frédéric SUR \\ LORIA - CNRS UMR 7503, INRIA, Université de Lorraine (France)
}

\begin{abstract}
Images can be affected by quasi-periodic noise. This undesirable feature manifests itself by spurious repetitive patterns covering the whole image, well localized in the Fourier domain. While notch filtering permits to get rid of this phenomenon, this however requires to first detect the resulting Fourier spikes, and, in particular, to discriminate between noise spikes and spectrum patterns caused by spatially localized textures or repetitive structures. This paper proposes a statistical a-contrario detection of noise spikes in the Fourier domain. A Matlab code is also provided.
\end{abstract}

Index Terms - quasi-periodic noise, a-contrario method.

\section{INTRODUCTION}

Images may be affected by quasi-periodic noise, that is, spurious repetitive patterns covering the entire image. This artifact is often caused by electrical interferences during image acquisition or transmission, which make remote sensing applications especially prone to the phenomenon. Periodic noise gives more or less sharp spikes in the image spectrum, which can be filtered out using notch filters. Some basic approaches requiring expert tuning can be found in $[1,2]$. The difficulty is to automate spike detection, that is, notch filter design. Some authors [3,4] suggest to detect spikes in the Fourier domain as large deviations with respect to a localized median value. However, distinguishing between spikes caused by a localized texture or a repetitive structure (common in man-made environments) and spurious ones caused by periodic noise is still challenging. It has been observed in [5] that periodic noise is likely to be the only periodic structure present in any patch extracted from the impaired image. It is proposed to design the notch filter based on the average power spectrum computed on a set of patches: the contribution of localized repetitive textures is smoothed out by averaging, and the only remaining spikes are the ones caused by periodic noise. We have recently automated this approach by detecting the spikes in the averaged spectrum as statistical outliers of the distribution expected from natural (non-noisy) patches, which is known to follow the inverse of a power of the frequency [6].

We propose to detect the Fourier spikes caused by periodic noise with an a-contrario method. A-contrario the-

This work is partially funded by GDR CNRS ISIS (TIMEX project). ory was introduced for the detection of alignments in images [7] and proves to be well adapted to many image analysis tasks as Gestalt grouping [8, 9], detection of moving objects in videos [10], sub-pixel change detection in satellite imagery [11], shape identification [12], line segment [13] or elliptical arc [14] detection, interest point matching [15, 16, 17, 18], point clustering [19], multiple object detection [20], or spot detection in textures [21], just to cite a few recent papers. This detection theory is based on the idea that features of interest (called meaningful features) are not likely to be caused by a random background process. Deciding whether a feature is meaningful or not is based on the number of false alarms (NFA) which corresponds to the average number of such a feature expected from the background process (hence "false alarms"). More precisely, in the case of real-valued features, if the features of interest are not likely to have a high value $x$, and provided that the meaningful features are sought among $N$ features, then the NFA of the observed $x$ is:

$$
\operatorname{NFA}(x)=N \operatorname{Pr}(X \geq x)
$$

where $\operatorname{Pr}(X \geq x)$ is the probability that a random feature $X$ following the background process is larger than $x$. In a-contrario models, $X$ is actually a function of independent variables (although recent papers explore nonindependence $[22,23]), \operatorname{Pr}(X>x)$ is thus calculated from marginal laws, which are either parametric [8] or empirically estimated [12]. Once an NFA has been defined, the feature of interest are in most papers those such that NFA $\leq 1$. This means that at most one such feature could be observed in the background model among the $N$ tested ones. In this sense, a-contrario detection is a parameter-free method. Psychophysical experiments suggest that a-contrario detection matches human perception [24, 25].

\section{A-CONTRARIO DETECTION OF SPECTRUM SPIKES CAUSED BY PERIODIC NOISE}

We adopt the same basic premise as in $[5,6]$ : considering a set of $P$ patches of size $L$ spanning the entire image, periodic noise is characterized by spikes present in the spectrum of every patch. In contrast, an image not affected by periodic noise does not show this feature, and localized periodic textures give Fourier spikes only in some of the patches. Our aim is thus to detect frequencies whose corresponding Fourier coefficients have an unexpected large amplitude in all 


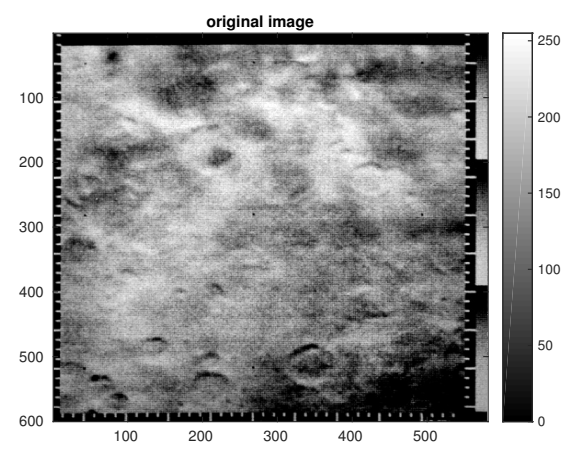

a)

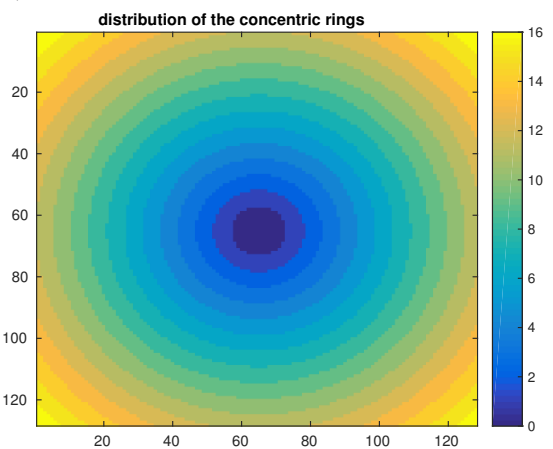

d)

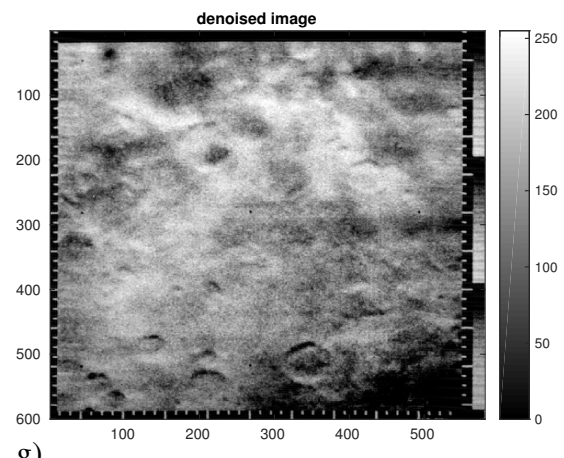

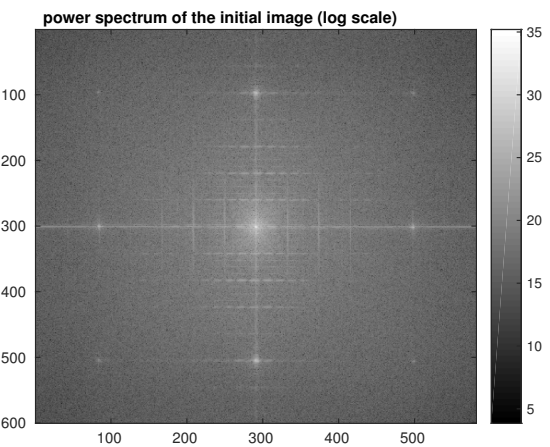

b)

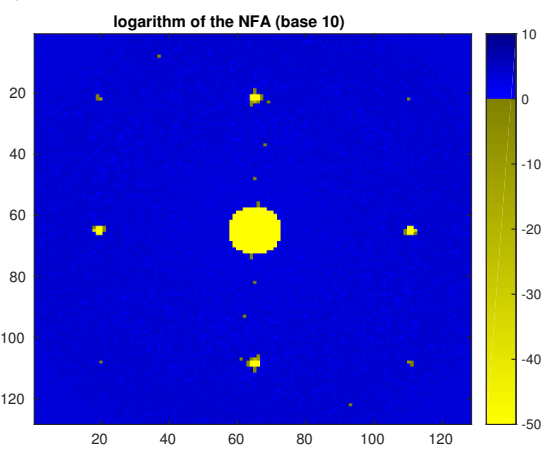

e)

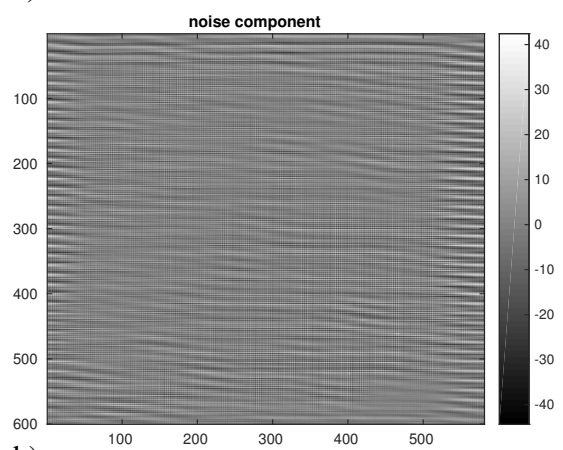

h)

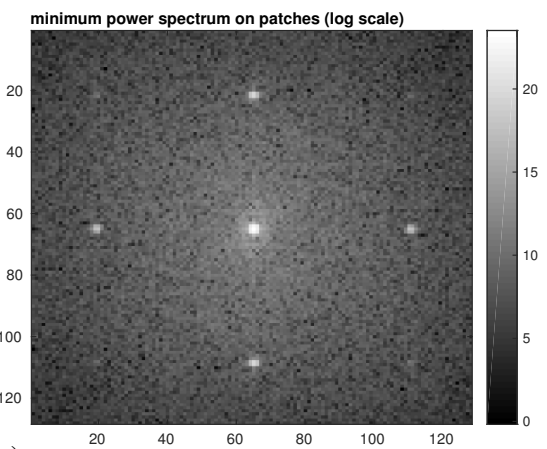

c)

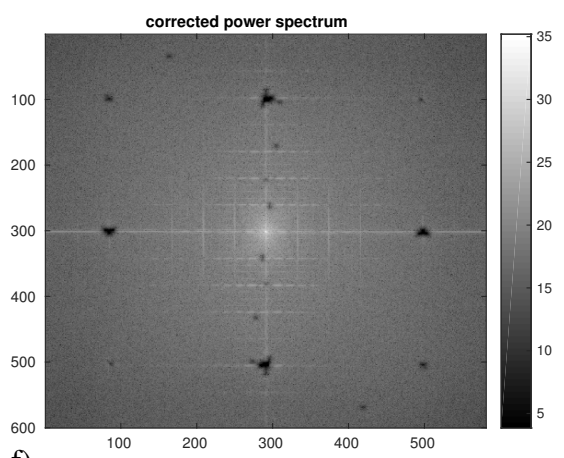

f)

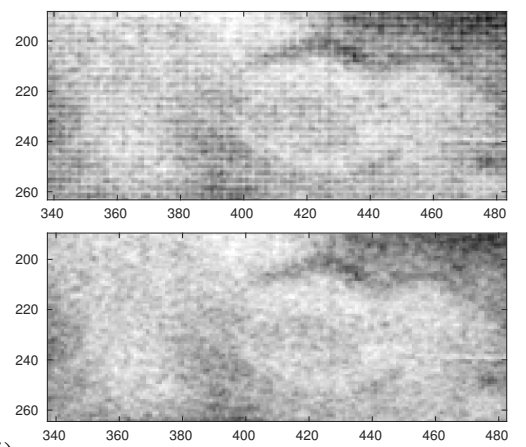

i)

Fig. 1. a) Original image; b) spectrum; c) element-wise minimum of the spectra of the $128 \times 128$ patches; d) distribution of the 16 concentric rings; e) map of the NFA; f) original power spectrum with the periodic noise spikes smoothed out; g) denoised image; h) retrieved noise component; i) comparison between close-ups of the original and denoised images.

patches, with an a-contrario approach. Let us consider the Fourier coefficient $c_{n, m}^{p}$ of frequency $(n, m)$ cycles per image of the $p$-th patch. We would like to characterize $(n, m)$ for which the minimum value $\min _{1 \leq p \leq P}\left|c_{n, m}^{p}\right|$ is not likely to be observed in a non-noisy image (here $|\cdot|$ is the complex modulus). In this case, there is a suspicious spike at $(n, m)$ in all of the $P$ patches. Let us define the background process for any $(n, m)$ as $X_{n, m}=\min _{1 \leq p \leq P}\left|C_{n, m}^{p}\right|$ with the $C_{n, m}^{p}$ independent and identically distributed random variables, their tail distribution being noted $F_{n, m}$. Because of the independence assumption, the probability in (1) writes

$$
\begin{aligned}
\operatorname{Pr}\left(\min _{1 \leq p \leq P}\left|C_{n, m}^{p}\right| \geq x\right) & =\operatorname{Pr}\left(\forall p,\left|C_{n, m}^{p}\right| \geq x\right) \\
& =\left(F_{n, m}(x)\right)^{P}
\end{aligned}
$$

The problem is now to design the distribution of the $C_{n, m}^{p}$ in the background model, i.e., for a non-noisy image. This distribution cannot obviously be chosen identical for all $(n, m)$ but should instead at least depend on the frequency $f=$ $\sqrt{n^{2}+m^{2}}$, the most striking evidence being the expected $1 / f$ power law of the spectrum [26]. We thus choose to cover the spectrum (centered at low frequencies) by $R=L / 8$ concentric rings, as in Fig. 1d), the distribution being fixed over a given ring but varying from ring to ring. Each distribution is empirically estimated from the Fourier coefficients of the $P$ patches belonging to the considered ring. That is, for any $x$,

$$
\begin{array}{r}
F_{\mathcal{R}(n, m)}(x)=\frac{1}{P \# \mathcal{R}(n, m)} \#\left\{\left(n^{\prime}, m^{\prime}, p\right)\right. \\
\text { s.t. } \left.\left|c_{n^{\prime}, m^{\prime}}^{p}\right| \geq x,\left(n^{\prime}, m^{\prime}\right) \in \mathcal{R}(n, m), 1 \leq p \leq P\right\}
\end{array}
$$


where \#. denotes the cardinality of any finite set, and $\mathcal{R}(n, m)$ denotes the ring to which $(n, m)$ belongs.

Note that it is easy to add an angular discretization to Fig. 1d) in order to satisfy anisotropic models as in [27].

In a given ring, only a small minority of coefficients may belong to a spike caused by periodic noise. It is thus valid to consider that $F_{\mathcal{R}(n, m)}(x)$ is the probability that $\left|C_{n, m}\right| \geq x$ for a non-noisy image, in accordance with the background process. The expected number of false alarms over a given ring $\mathcal{R}$ (where the tail distribution is $F_{\mathcal{R}}$ ) is thus $\# \mathcal{R} \cdot F_{\mathcal{R}}(x)$. Since there are $R$ such rings,

$$
\left.\operatorname{NFA}\left(\left|c_{n, m}\right|\right)\right)=R \# \mathcal{R}(n, m)\left(F_{\mathcal{R}(n, m)}\left(\left|c_{n, m}\right|\right)\right)^{P}
$$

is a valid definition for a number of false alarms (see Def. 4 and Prop. 2 in [21]), in the sense that it satisfies:

Proposition The expected number of random spectrum coefficients following the background process with an NFA below $\varepsilon$ is smaller than $\varepsilon$, i.e.,

$$
E\left(\#\left\{(n, m) \text { s.t. } N F A\left(\left|C_{n, m}\right|\right) \leq \varepsilon\right\}\right) \leq \varepsilon
$$

Consequently, frequencies $(n, m)$ such that $\min _{p} c_{n, m}^{p}$ has an NFA below 1 are not likely to come from a non-noisy image, and are confidently considered as coming from periodic noise.

Remark 1 A-contrario detection of suspicious spectrum structures is also used in [28] for image aliasing and in [29] for motion blur, without, however, any spectrum model.

Remark 2 In [5, 6], noise spikes are detected from an average power spectrum. With the notation of (2)-(3), the distribution of $X_{n, m}=\frac{1}{P} \sum_{p=1}^{P}\left|C_{n, m}^{p}\right|$ is a scaled $P$-fold convolution of the distribution of $\left|C_{n, m}\right|$ with itself. An NFA could thus be defined similarly to (2)-(3), in the same spirit as in [18]. It could benefit from the central limit theorem by approximating the distribution of $X_{n, m}$ for large $P$ by a normal distribution, of mean value $\simeq 1 / f^{\gamma}$. Similarly, the empirical $F_{n, m}^{P}$ in (3) could be replaced with a parametric distribution such as a Gumbel, Fréchet or Weibull distribution, following the extreme value theory [30].

\section{QUASI-PERIODIC NOISE REMOVAL}

Spikes caused by periodic noise are detected by thresholding the map of the $\operatorname{NFA}\left(\left|c_{n, m}\right|\right)$ to 1 . Based on these spikes, the same strategy as in [6] is employed: the spikes are propagated to the original image spectrum and permits to build a notch filter which smooths out the contribution of the periodic noise. Given an image impaired by quasi-periodic noise, the proposed algorithm is described in Alg. 1. We use the same implementation details as in [6] where a thorough discussion of the parameters can also be found.

Fig. 1 shows a running example. a) is an image obtained by the Mariner 4 probe, affected by a quasi-periodic noise, and $b$ ) is its power spectrum. Visually discriminating the noise components in the spectrum is not obvious. c) shows the minimum of the power spectra from the patches (step 2, here $L=128$ pixels). Four spikes are clearly visible, in
Algorithm 1 A-contrario quasi-periodic noise removal Input: image $i$ (size $X \times Y$, Fourier transform $I$ ), patch size $L$.

1. Extract non-overlapping patches (to enforce the independence assumption in (2)-(3)) of size $L \times L$ distributed over $i$ (giving $P$ patches) and calculate the $F_{\mathcal{R}}$ with (4).

2. Calculate the minimum of the power spectra of the patches: for any $(n, m),\left|c_{n, m}\right|=\min _{p=1 \ldots P}\left|c_{n, m}^{p}\right|$.

3. For any $(n, m)$, calculate NFA $\left(\left|c_{n, m}\right|\right)$ with (5).

4. Define the spike map $M_{o}^{\mathcal{P}}$ on the $L \times L$ spectrum such that $M_{o}^{\mathcal{P}}(n, m)=1$ if $\operatorname{NFA}\left(\left|c_{n, m}\right|\right) \leq 1$, and 0 otherwise.

5. Interpolate the outlier map $M_{o}^{\mathcal{P}}$ of size $L \times L$ to $X \times Y$, giving a map $M_{o}$ of the probable spurious spikes in the original image spectrum. Multiplying the initial image spectrum by $1-M_{o}$ acts as a notch filter, eliminating the influence of the quasi-periodic noise.

6. Retrieve $\widehat{n}$, estimation of the periodic noise component, as the inverse Fourier transform (IFT) of $M_{o} I$, and $\widehat{i}$, estimation of the denoised image, as $i-\widehat{n}$ (i.e., IFT of $\left.\left(1-M_{o}\right) I\right)$.

addition to the central low-frequency component. d) shows the distribution of the $R=L / 8=16$ concentric rings on which the Fourier coefficient amplitudes are assumed to be identically distributed. The central disk (in deep blue) is not taken into account by the algorithm, because low frequencies do not correspond to repetitive patterns (moreover, the identical distribution hypothesis is not valid in this disk). e) is the map of the logarithm of the NFA (with an arbitrary value in the central disk): a large majority of the coefficients give an NFA larger than $1(\log (\mathrm{NFA})>0)$, except for the four spikes with a very low NFA $\left(\simeq 10^{-180}\right)$. f) is the corrected spectrum $\left(1-M_{o}\right) I$ (step 5): we can see that, in addition to the four spikes, a few other coefficients have been detected as meaningful (with NFA $\simeq 10^{-1}$ ). g) is $\widehat{i}$, and h) is $\widehat{n}$ (step 6). Real image details cannot be seen in $\widehat{n}$, which suggests that the noise component has been well separated from the actual data. i) shows close-ups of $i$ and $\widehat{i}$.

\section{EXPERIMENTS}

Some results are presented in Fig. 2. First, a synthetic periodic noise has been added to the Mandrill image. The underlying image and the noise component are well separated by our algorithm. This shows that the proposed a-contrario detection is able to detect the noise spikes even in the presence of a strong high-frequency texture (the fur here) affecting most patches. The two remaining experiments deal with real images. The Lunar Orbiter image is impaired by striping, which is adequately removed. The Mariner 6 image is affected by noise patterns whose spectrum is much more complex than just a few separated spikes. The algorithm is still able to separate the noise component from the image.

Additional experiments and a Matlab code are available at: www.loria.fr/\%7Esur/software/ACARPENOS/ 

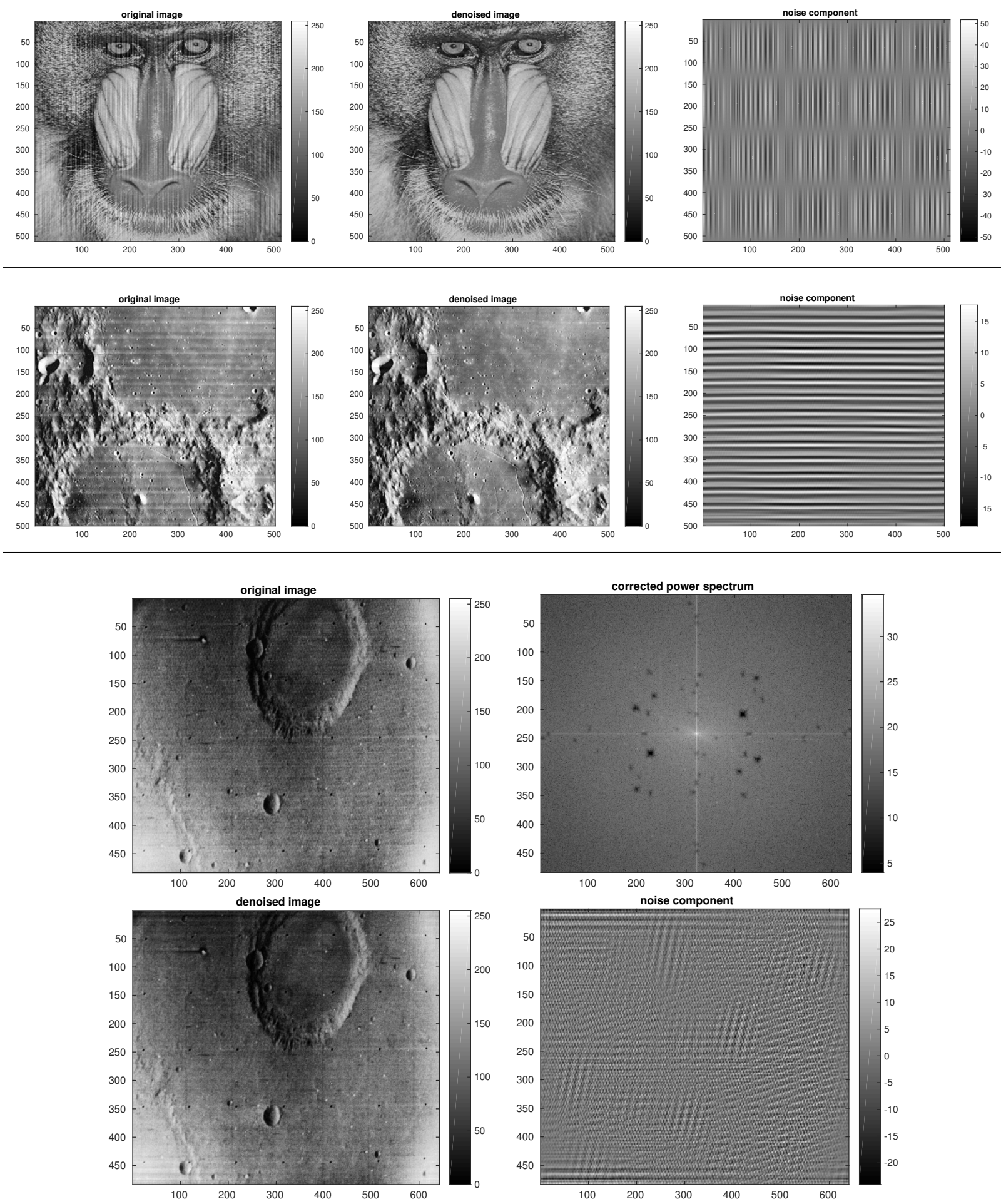

Fig. 2. From top to bottom: Mandrill, Lunar Orbiter, and Mariner 6 experiments. The reader is asked to zoom in on the pdf file.

\section{CONCLUSION}

This paper discussed a new a-contrario detection of the spectrum components of a quasi-periodic noise, making it possible to design notch filters for periodic noise removal. While ex- isting similar methods $[5,6]$ use an average power spectrum calculated from image patches, the proposed method is based on the minimum spectrum and benefits from the parameterfree decision method offered by the a-contrario approach. 


\section{REFERENCES}

[1] R.C. Gonzalez and R.E. Woods, Digital image processing, Prentice-Hall, 2006.

[2] M. Grédiac, F. Sur, and B. Blaysat, "Removing quasiperiodic noise in strain maps by filtering in the Fourier domain," SEM Exp. Tech., 2015, to be published.

[3] I. Aizenberg and C. Butakoff, "A windowed Gaussian notch filter for quasi-periodic noise removal," Image Vision Comput., vol. 26, no. 10, pp. 1347-1353, 2008.

[4] G.A.A. Hudhud and M.J. Turner, "Digital removal of power frequency artifacts using a Fourier space median filter," IEEE Signal Process. Lett., vol. 12, no. 8, pp. 573-576, 2005.

[5] M. Cannon, A. Lehar, and F. Preston, "Background pattern removal by power spectral filtering," Appl. Optics, vol. 22, no. 6, pp. 777-779, 1983.

[6] F. Sur and M. Grédiac, "Automated removal of quasiperiodic noise using frequency domain statistics," $I S \& T$ / SPIE J. Electron. Imaging, vol. 24, no. 1, pp. 013003/1-19, 2015.

[7] A. Desolneux, L. Moisan, and J.-M. Morel, "Meaningful alignments," Int. J. Comput. Vision, vol. 40, no. 1, pp. 7-23, 2000.

[8] A. Desolneux, L. Moisan, and J.-M. Morel, From Gestalt theory to image analysis: a probabilistic approach, Springer, 2008.

[9] J. Lezama, R. Grompone von Gioi, G. Randall, and J.M. Morel, "A contrario detection of good continuation of points," in Proc. ICIP, 2014.

[10] F. Dibos, G. Koepfler, and S. Pelletier, "Adapted windows detection of moving objects in video scenes," SIAM J. Imaging Sci., vol. 2, no. 1, pp. 1-19, 2009.

[11] A. Robin, L. Moisan, and S. Le Hegarat-Mascle, "An a-contrario approach for subpixel change detection in satellite imagery," IEEE Trans. Pattern Anal. Mach. Intell., vol. 32, no. 11, pp. 1977-1993, 2010.

[12] F. Cao, J.L. Lisani, J.-M. Morel, P. Musé, and F. Sur, $A$ theory of shape identification, Springer, 2008.

[13] R. Grompone von Gioi, J. Jakubowicz, J.-M. Morel, and G. Randall, "LSD: a fast line segment detector with a false detection control," IEEE Trans. Pattern Anal. Mach. Intell.,, vol. 32, no. 4, pp. 722-732, 2010.

[14] V. Patraucean, P. Gurdjos, and R. Grompone von Gioi, "A parameterless line segment and elliptical arc detector with enhanced ellipse fitting," in Proc. ECCV, 2012.

[15] N. Noury, F. Sur, and M.-O. Berger, "How to overcome perceptual aliasing in ASIFT?," in Proc. ISVC, 2010.
[16] F. Sur, N. Noury, and M.-O. Berger, "An a contrario model for matching interest points under geometric and photometric constraints," SIAM J. Imaging Sci., vol. 6, no. 4, pp. 1956-1978, 2013.

[17] L. Moisan and B. Stival, "A probabilistic criterion to detect rigid point matches between two images and estimate the fundamental matrix," Int. J. Comput. Vision, vol. 57, no. 3, pp. 201-218, 2004.

[18] J. Rabin, J. Delon, and Y. Gousseau, "A statistical approach to the matching of local features," SIAM J. Imaging Sci., vol. 2, no. 3, pp. 931-958, 2009.

[19] M. Tepper, P. Musé, A. Almansa, and M. Mejail, "Automatically finding clusters in normalized cuts," Pattern Recognit., vol. 44, no. 7, pp. 1372-1386, 2011.

[20] J. Rabin, J. Delon, Y. Gousseau, and L. Moisan, "MACRANSAC: a robust algorithm for the recognition of multiple objects," in Proc. 3DPVT, 2010.

[21] B. Grosjean and L. Moisan, "A-contrario detectability of spots in textured backgrounds," J. Math. Imaging Vision, vol. 33, no. 3, pp. 313-337, 2009.

[22] A. Myaskouvskey, Y. Gousseau, and M. Lindenbaum, "Beyond independence: an extension of the a contrario decision procedure," Int. J. Comput. Vision, vol. 101, no. 1, pp. 22-44, 2013.

[23] A. Flenner and G. Hewer, "A Helmholtz principle approach to parameter free change detection and coherent motion using exchangeable random variables," SIAM J. Imaging Sci., vol. 4, no. 1, pp. 243-276, 2011.

[24] S. Blusseau, A. Carboni, A. Maiche, J.-M. Morel, and R. Grompone von Gioi, "A psychophysical evaluation of the a contrario detection theory," in Proc. ICIP, 2014.

[25] A. Desolneux, L. Moisan, and J.-M. Morel, "Computational gestalts and perception thresholds," $J$. Physiology-Paris, vol. 97, no. 23, pp. 311-324, 2003.

[26] D.J. Field, "Relations between the statistics of natural images and the response properties of cortical cells," $J$. Opt. Soc. Amer. A, vol. 4, pp. 2379-2394, 1987.

[27] A. Oliva and A. Torralba, "Modeling the shape of the scene: a holistic representation of the spatial envelope," Int. J. Comput. Vision, vol. 42, no. 3, pp. 145-175, 2001.

[28] B. Coulange and L. Moisan, "An aliasing detection algorithm based on suspicious colocalizations of Fourier coefficients," in Proc. ICIP, 2010.

[29] F. Xue, Q. Liu, and J. Froment, "An a contrario approach for parameters estimation of a motion-blurred image," in Proc. EMMCVPR, 2007.

[30] S. Coles, An introduction to statistical modeling of extreme values, Springer, 2001. 